\title{
Aplicações da telemedicina no cenário da pandemia de COVID-19
}

\author{
Telemedicine applications in the COVID-19 pandemic scenario \\ Aplicaciones de la telemedicina en el escenario de la pandemia de COVID-19
}

Recebido: 17/12/2021 | Revisado: 27/12/2021 | Aceito: 29/12/2021 | Publicado: 07/01/2022

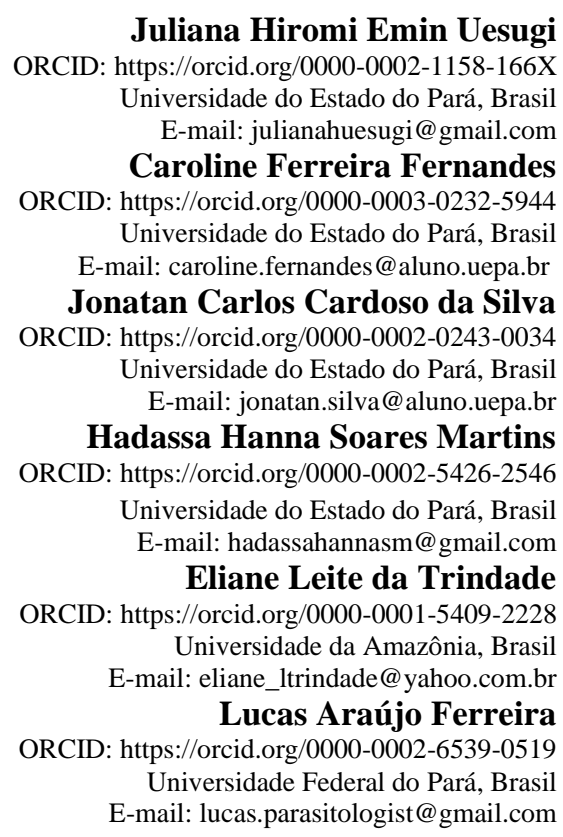

\section{Resumo}

O objetivo deste trabalho foi descrever as aplicações da telemedicina no atual cenário da pandemia de COVID-19 com enfoque no Brasil. Trata-se de um estudo descritivo, qualitativo, realizado a partir de um levantamento bibliográfico acerca dos impactos positivos e negativos do uso da telemedicina na atualidade. A literatura foi obtida das plataformas SciELO, ResearchGate, PubMed e World Wide Science. A telemedicina pode se tornar uma ferramenta fundamental para garantir a universalização da saúde, pois possibilita que consultas e atendimentos especializados sejam realizados de maneira remota, reduzindo custos com deslocamento e ampliando o acesso à saúde pela população. Com as medidas de segurança impostas com a pandemia de SARS-CoV-2, a telessaúde se tornou uma opção viável, pois evita a exposição de indivíduos ao vírus nas unidades de saúde. Apesar disso, existem desafios relacionados à sua utilização, como o alto custo, a resistência de profissionais de saúde e pacientes, as desigualdades regionais e a falta de profissionais qualificados. Portanto, cabe refletir acerca da aplicabilidade da telemedicina no atual cenário brasileiro. Embora existam algumas dificuldades quanto à adesão da telemedicina no país, é evidente que ela traz muitos benefícios para os sistemas de saúde. Com o aumento dos investimentos em todas as regiões brasileiras de acordo com as suas necessidades, a telemedicina poderá solucionar desafios recorrentes no sistema de saúde, a partir da ampliação da cobertura de serviços especializados, melhoria da qualidade da atenção, redução do tempo de diagnóstico e terapia e diminuição de custos com deslocamento.

Palavras-chave: Telemedicina; Telessaúde; Saúde pública.

\begin{abstract}
This study aims to describe the applications of telemedicine in the current scenario of the COVID-19 pandemic focusing in Brazil. This is a descriptive, qualitative study, carried out from a bibliographic survey about the positive and negative impacts of the use of telemedicine today. The literature was obtained from the SciELO, ResearchGate, PubMed and World Wide Science platforms. Telemedicine can become a fundamental tool to ensure universal access to health. It enables consultations and specialized care to be carried out remotely, reducing displacement costs and expanding access to healthcare by the population. With the security measures imposed with the SARS-CoV-2 pandemic, telehealth has become a practicable option to prevent the population from being exposed to the virus in the healthcare units. Despite that, there are challenges related to its use, such as the high cost, the resistance from health professionals and patients, regional inequalities and the lack of qualified professionals. Therefore, it is important to reflect on the applicability of telemedicine in the current Brazilian scenario. Although there are some difficulties
\end{abstract}


regarding the adoption of telemedicine in our country, it is evident that it brings many benefits to the health systems. With the increase in investments in all Brazilian regions according to their needs, telemedicine will be able to solve recurrent challenges in the health system, by expanding the coverage of specialized services, improving the quality of care, reducing the time for diagnosis and therapy and cost reduction with displacement.

Keywords: Telemedicine; Telehealth; Public health.

\section{Resumen}

El objetivo de este estudio fue describir el uso y las aplicaciones de la telemedicina en el escenario actual de la pandemia de COVID-19, centrándose en Brasil. Se trata de un estudio descriptivo y cualitativo, realizado a partir de una encuesta bibliográfica sobre los impactos positivos y negativos del uso de la telemedicina en la actualidad. La literatura se obtuvo de las plataformas SciELO, ResearchGate, PubMed y World Wide Science. La telemedicina puede convertirse en una herramienta fundamental para garantizar la atención sanitaria universal, ya que permite realizar consultas y atención especializada a distancia, reduciendo los costes de desplazamiento y ampliando el acceso de la población a la atención sanitaria. Con las medidas de seguridad impuestas por la pandemia de SARS-CoV-2, la telesalud se ha convertido en una opción viable, ya que evita la exposición de los individuos al virus en las unidades sanitarias. Sin embargo, existen retos relacionados con su uso, como su elevado coste, la resistencia de los profesionales sanitarios y los pacientes, las desigualdades regionales y la falta de profesionales cualificados. Por lo tanto, vale la pena reflexionar sobre la aplicabilidad de la telemedicina en el escenario brasileño actual. Aunque existen algunas dificultades para la adopción de la telemedicina en el país, está claro que aporta muchos beneficios a los sistemas sanitarios. Con el aumento de las inversiones en todas las regiones brasileñas de acuerdo con sus necesidades, la telemedicina puede resolver retos recurrentes en el sistema de salud, desde la ampliación de la cobertura de los servicios especializados, la mejora de la calidad de la atención, la reducción del tiempo para el diagnóstico y la terapia y la reducción de los costos de viaje.

Palabras clave: Telemedicina; Telesalud; Salud pública.

\section{Introdução}

O Sistema Único de Saúde (SUS) foi criado com objetivo de garantir o acesso universal e igualitário aos serviços de saúde, atuando de acordo com diretrizes e princípios éticos. Sendo assim, o acesso à saúde pelos brasileiros deve ser comum para todos, sem que haja privilégios a grupos específicos em detrimento de outros, como distinção entre classes sociais, etnia, moradia, sexo, etc (Brasil, 1990).

A telemedicina consiste na aplicação de tecnologias de informação na saúde com objetivo de ampliar a oferta de serviços básicos, principalmente quando a distância entre o paciente e o centro de saúde procurado é um fator crítico. Ela surgiu como uma alternativa complementar à medicina tradicional, com o objetivo de facilitar e ampliar a cobertura dos serviços de saúde (Maldonado, 2016; Zanotto, 2020).

Existem três dimensões da telemedicina: a funcionalidade, que inclui os serviços para os quais ela é utilizada (consultas, diagnósticos, acompanhamento), a tecnologia, que trata da sincronização, da rede e da conectividade necessários (podendo usar metodologias de atendimento em tempo real ou em tempo diferido) e a aplicação, que se refere aos processos assistenciais, como as especialidades médicas, locais de atendimento e tratamento individual (Cha Gligia, 2020).

Nos últimos anos, criou-se condições favoráveis à implementação da telemedicina no país, como o envelhecimento da população e o consequente aumento na prevalência de doenças crônicas, as quais precisam de monitoramento contínuo. Além disso, a telemedicina abrange múltiplas áreas de atendimento, como teleconsulta, telerradiologia, teledermatologia, telepatologia e telepsiquiatria. Sendo assim, a telemedicina permite que esses e outros pacientes tenham acesso aos centros de atendimento especializado sem necessidade de locomoção, possibilita a resolução de problemas a nível de atenção básica e facilita o acompanhamento da condição do paciente (Wen, 2015; Paula, 2020; WHO, 2020).

Com o advento da pandemia causada pelo novo Coronavírus (SARS-CoV-2), novos desafios surgiram de forma imprevisível para a população mundial, pois demandou uma nova organização em relação às rotinas, lazer, trabalho, consumo e utilização de serviços, principalmente os de saúde. Mediante esse cenário, a telemedicina se demonstrou crucial, tendo em vista a ampliação do acesso à saúde pelos habitantes de regiões mais remotas sem haver necessidade de deslocamento e exposição à doença (Bhaskar, 2020; Dimer, 2020). 
Mediante a alta transmissibilidade da doença COVID-19, a legislação de $n^{\circ}$ 13.989/2020 dispôs sobre a utilização da telemedicina diante da crise proposta pela emergência de saúde pública. Assim, a telessaúde constitui um componente importante ao controle da disseminação do coronavírus, pois permite a redução da circulação de indivíduos às unidades da saúde, reduzindo a exposição dos pacientes, mantendo a qualidade e segurança dos serviços concomitantemente (Brasil, 2020; Caetano, 2020).

Apesar dessas vantagens, ainda há controvérsias com relação ao uso desse serviço no país, uma vez que a incorporação da telemedicina à prática médica atual requer um equilíbrio entre qualidade e eficiência, além de investimentos em infraestrutura. Em algumas regiões do Brasil, a falta de um orçamento suficiente para a implantação e manutenção dos sistemas de telessaúde, de infraestrutura e a ausência de parâmetros legais para essa prática dificulta com que seja efetivamente adotada (Sabbatini, 2012; Zanaboni, 2014). Assim, tendo em vista as características inerentes à prática da telemedicina, principalmente no contexto emergencial dado à pandemia causada pelo SARS-CoV-2, o presente estudo veio por descrever a utilização da telemedicina com enfoque na realidade do Brasil.

\section{Metodologia}

\section{Tipo de Estudo e bases de dados}

Trata-se de um estudo descritivo, qualitativo, realizado a partir de um levantamento bibliográfico acerca dos impactos positivos e negativos do uso da telemedicina na atualidade. Para tal a literatura utilizada foi obtida das bases de dados: Scientific Eletronic Library Online (SciELO), ResearchGate, U.S National Institutes of Health's Library of Medicine (PubMed) e World Wide Science. A pesquisa foi realizada entre os meses de junho e dezembro de 2021.

\section{Descritores}

Para a realização do estudo, foram utilizados os seguintes descritores nos idiomas português, inglês e espanhol: telessaúde/telehealth/telesalud, telemedicina/telemedicineltelemedicina e COVID-19.

\section{Critérios de inclusão e exclusão}

Adotou-se como critérios de inclusão: artigos publicados entre o período de 01 de janeiro de 2010 a 01 de dezembro de 2021; trabalhos publicados nos idiomas de português, inglês e espanhol; trabalhos que abordassem o tema de telessaúde e que tratassem das aplicações de telemedicina no contexto da pandemia de COVID-19. Os critérios de exclusão foram: artigos publicados fora do período delimitado; trabalhos apresentados em congressos; trabalhos de conclusão de curso, capítulos de livro ou que não abordassem a temática estabelecida.

\section{Resultados e Discussão}

A busca ativa resultou em 41 artigos encontrados nos idiomas de português, inglês ou espanhol, dentre os quais 27 foram selecionados para segunda análise pelos autores e 13 foram escolhidos pelos autores por atender aos critérios préestabelecidos neste trabalho (Figura 1). 
Figura 1: Pesquisa e seleção de artigos para a realização desta revisão com base nos critérios pré-estabelecidos pelos autores.

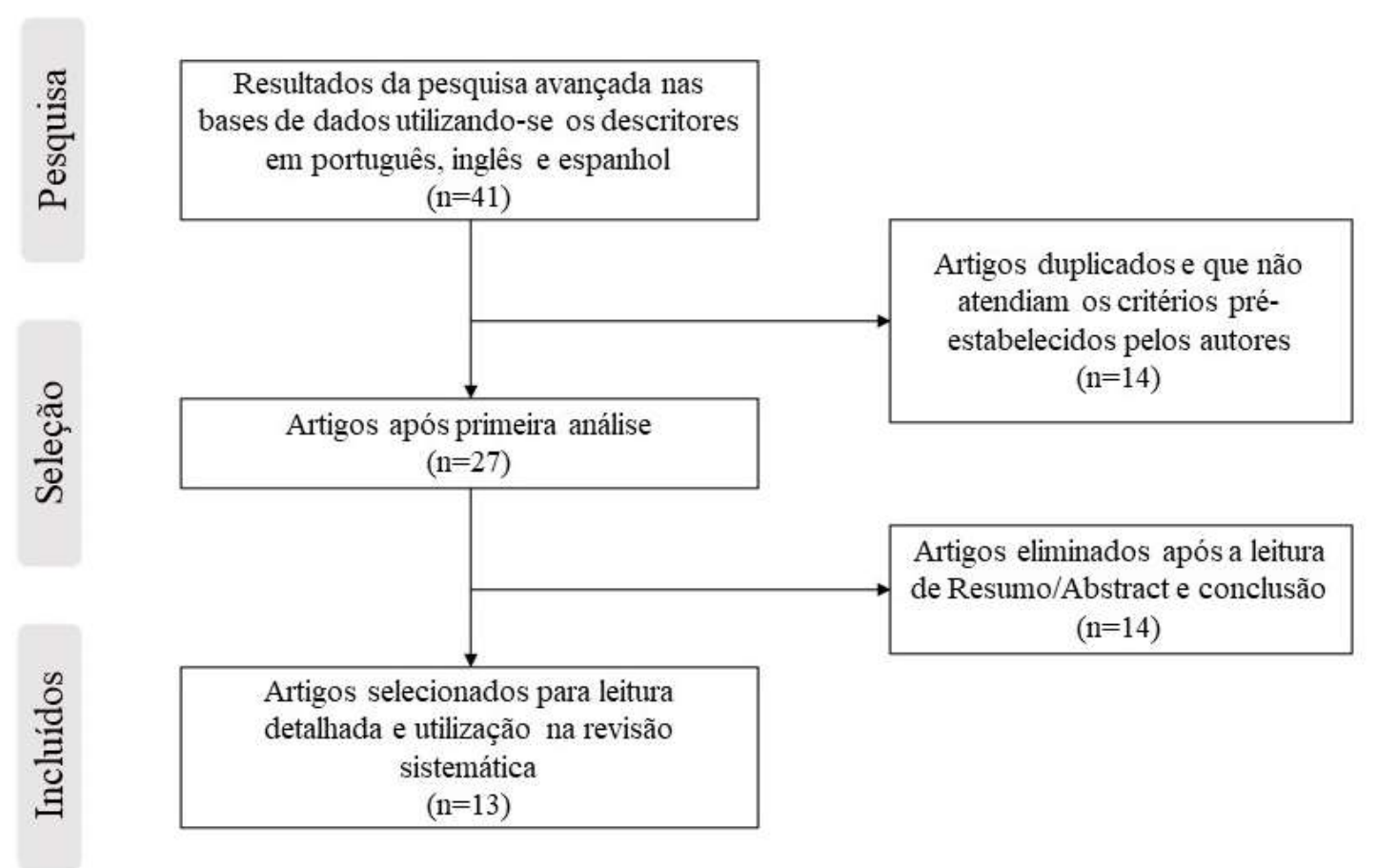

Fonte: Autores. Adaptado de Cruz et al, (2021).

O trabalho de Machado et al. (2010), aborda a necessidade de implementar a telemedicina no país, uma vez que a distância, o elevado custo de transportes, a falta de recursos e de profissionais de saúde em comunidades mais distantes isoladas impede o acesso igualitário aos serviços de saúde. Ainda assim, os autores ressaltam que a telemedicina não é uma alternativa para substituir totalmente a prática médica tradicional, e sim para atuar de maneira integrada e sinérgica possibilitando o melhor atendimento possível aos pacientes.

Mattos e colaboradores (2015) que ao utilizar os recursos da telemedicina, os períodos de hospitalização e espera podem ser reduzidos, uma vez que ela permite um melhor acompanhamento e manejo dos pacientes crônicos sem necessidade de locomoção. A questão da desigualdade de acesso à saúde é enfatizada no trabalho de Almeida (2017), no qual o autor relata uma grande precariedade das condições socioeconômicas, de oferta e complexidade dos serviços de saúde nas regiões Norte e Nordeste, associando-as à falta de planejamento das necessidades da saúde da população. Situação enfatizada por Lopes, Oliveira e Maia (2019), onde relatam a diferença significativa entre postos de saúde de cada região, principalmente se comparar as regiões Norte e Sul do país, pois nas regiões mais distantes das metrópoles, observaram que grande parte das UBS não possuíam infraestrutura mínima para a aplicação desse serviço, além de haver dificuldades regionais e de acesso às tecnologias de informação.

Luz (2019) aponta que ainda há grande dificuldade dos médicos e principalmente dos pacientes se sentirem seguros no compartilhamento de informações pessoais por meio de plataformas remotas e também à adaptação às mudanças estruturais no método de atendimento e na rotina. Entretanto, Brito e Leitão (2020) lembram que a telemedicina pode ser efetiva e possibilitar a inclusão de populações de áreas mais remotas, como os ribeirinhos, garantindo educação, promoção à saúde e atendimento especializado. Tal qual dito por Castro e Alanis (2020) onde a telessaúde seria uma estratégia viável para atender as populações mais remotas e vulneráveis com menor acesso à saúde, embora ainda haja indivíduos que não conseguem acessá-la por falta de aparelhos, internet e conhecimento sobre as tecnologias de informação. 
No trabalho de Carvalho e colaboradores (2020) é afirmado que a telemedicina no contexto da pandemia de COVID19 possibilitou além os atendimentos à distância, o treinamento de profissionais de saúde, como médicos, fisioterapeutas e enfermeiros, além de possibilitar uma rede de monitoramento de pacientes em Unidades de Terapia Intensiva por equipes multiprofissionais, aprimorando a qualidade de atendimento especializado aos pacientes.

Os trabalhos de Santos (2020) e Catapan et al. (2021) apontam obstáculos quanto à utilização da telemedicina no país, como a dificuldade de criar laços de confiança entre médico e paciente, o conhecimento ainda incerto acerca das possibilidades de haver uma elevação na quantidade de erros médicos com o uso dessa técnica, o alto custo de implantação, a resistência dos pacientes e dos profissionais de saúde a usarem, a falta de profissionais qualificados e adaptados a essa metodologia, as desigualdades regionais e a carência de investimentos governamentais para o uso das tecnologias de saúde.

Ao analisar a aplicação das tecnologias de informação na situação da pandemia de COVID-19, Silva e colaboradores (2021) enfatizam que graças a articulações de múltiplas ações, como teleconsulta, telemonitoramento e telediagnóstico, é possível reduzir muitos encaminhamentos desnecessários, utilização excessiva de recursos tecnológicos e também diminuir a circulação e exposição de pessoas ao vírus.

Castro et al. (2021), afirmam que apesar das controvérsias associadas à relação médico-paciente na prática da telemedicina, é possível manter as consultas de maneira remota sem perder o fator humano e com respeito à dignidade de cada paciente, o que permitirá com que seu uso seja continuado mesmo após o período da pandemia. Além disso, a pandemia de SARS-CoV-2 denotou grande visibilidade e evolução da telemedicina, como abordado na revisão realizada por Kieling e colaboradores (2021), pois o contexto emergencial enfrentado possibilitou o aprofundamento as pesquisas relacionadas ao teleatendimento, além de contribuir para a disseminação de informações e serviços à várias regiões de saúde.

\section{Conclusão}

É possível compreender que a telemedicina, para os diferentes autores, pode apresentar fatores positivos e negativos, a considerar as questões éticas, econômicas, políticas e propriamente de saúde, mas que devido a pandemia causada pelo SARSCoV-2 trouxe à tona a necessidade de um sistema de telessaúde eficaz pudesse atender as demandas de toda a população. Tendo em vista que a utilização da telemedicina era prevista pela Lei $n^{\circ}$ 13.989/2020, foi possível a manutenção de grande parte dos atendimentos básicos e especializados, reduzindo, assim, a exposição dos indivíduos ao vírus e disseminação da doença. Ainda assim, foi possível observar que ainda há grandes desigualdades regionais que dificultam a instalação da telemedicina em todas as localidades do país.

Embora existam algumas dificuldades quanto à adesão e a implantação da telemedicina no país, é evidente que ela traz muitos benefícios para o sistema de saúde público. Com o aumento dos investimentos em equipamentos e em capacitações para os profissionais de saúde nesse âmbito, a telemedicina poderá solucionar desafios recorrentes no sistema de saúde, a partir da ampliação da cobertura de serviços especializados para todas as regiões, a melhoria da qualidade da atenção, redução do tempo de diagnóstico e terapia, diminuição de custos com deslocamento e de exposições desnecessárias e a facilidade em realizar o acompanhamento clínico efetivo de pacientes com enfermidades crônicas.

Para que a telemedicina seja efetivamente implantada na atenção básica, os governantes, profissionais de saúde e as autoridades devem contribuir com o monitoramento e com ações políticas eficazes com relação à utilização da telessaúde em todos os níveis de assistência e investir para que esse sistema esteja disponível igualmente em todo o território brasileiro. Além disso, é importante que os profissionais de saúde estejam devidamente qualificados para atender dentro dessa nova metodologia, informando-se acerca dos seus benefícios e de novas abordagens para transmitir segurança e confiança aos pacientes. 


\section{Referências}

Almeida, M. W. (2017). Desigualdade social e em saúde no Brasil: a telemedicina como instrumento de mitigação em João Pessoa-PB. J Bras Econ Saúde, 9 (3): 292-303.

Bhaskar, S, Bradley, S, Chattu, V. K. et al (2020). Telemedicine Across the Globe-Position Paper From the COVID-19 Pandemic Health System Resilience PROGRAM (REPROGRAM) International Consortium (Part 1). Front Public Health.

Brasil. (2020). Lei 13.989/20. Dispõe sobre o uso da telemedicina durante a crise causada pelo coronavírus (SARS-CoV-2). 2020. https://www.in.gov.br/en/web/dou/-/lei-n-13.989-de-15-de-abril-de-2020-252726328.

Brasil. (1990). Lei 8080/90. Dispõe sobre as condições para a promoção, proteção e recuperação da saúde, a organização e o funcionamento dos serviços correspondentes e dá outras providências. 1990. https://conselho.saude.gov.br/legislacao/lei8080_190990.htm.

Brito, B. O. \& Leitão, L. P. C. (2020). Telemedicina no Brasil: Uma estratégia possível para o cuidado em saúde em tempo de pandemia? Saúde em Redes, 6(Supl.2). 10.18310/2446- 48132020v6n2Suplem.3202g550

Caetano, R, Silva, A. B. \& Guedes, A. C. C. M. et al. (2020). Desafios e oportunidades para telessaúde em tempos da pandemia pela COVID-19: uma reflexão sobre os espaços e iniciativas no contexto brasileiro. Cadernos de Saúde Pública. v. 36, n., e00088920. https://doi.org/10.1590/0102-311X00088920

Carvalho, C. R. R, Scudeller, P. G., Rabello, G. et al. (2020). Use of telemedicine to combat the COVID-19 pandemic in Brazil. CLINICS. $10.6061 /$ clinics/2020/e2217

Castro, A. T., Álvarez, D. C. \& Alanís, J. C. S. (2021). Telemedicina em Tiempos de Pandemia. Acta Med Grupo Angeles. 19 (s1): s80-s83. https://dx.doi.org/10.35366/101034.

Castro, F. A. G., Santos, A. O., Reis, G. V. L. et al. (2020). Telemedicina rural e COVID-19: ampliando o acesso onde a distância já era regra. Rev Bras Med Fam Comunidade. Rio de Janeiro, 15(42):2484.

Catapan, S. C., Willemann, M. C. A. \& Calvo, M. C. M. (2021). Estrutura e processo de trabalho para implantação da teleconsulta médica no Sistema Único de Saúde do Brasil, um estudo transversal com dados de 2017-2018. Epidemiologia e Serviços de Saúde, v. 30 , p. e2020305.

Cha Ghiglia, M. M. (2020). Telemedicina: su rol en las organizaciones de salud. Rev. Méd. Urug., Montevideo, 36(4), 185203. http://www.scielo.edu.uy/scielo.php?script=sci_arttext\&pid=S1688-03902020000400185\&lng=es\&nrm=iso.

Cruz, M. G. C., Cavalcanti, L. A. M., D’Albuquerque, A. C. et al. (2021). Vantagens e entraves do uso da telemedicina no acompanhamento de pacientes com insuficiência cardíaca. Research, Society and Development, 10(9), e0410917708. http://dx.doi.org/10.33448/rsd-v10i9.17708

Kieling, D. L., Silva, D. L., Witt, F. M et al. (2021). The importance of telemedicine in the COVID-19 pandemic contex. FAG Journal of Health, 3(1), 90-97. https://doi.org/10.35984/fjh.v3i1.302.

Lopes, M. A. C. Q., Oliveira, G. M. M. \& Maia, L. M. (2019). Saúde digital, direito de todos, dever do Estado? Arquivos Brasileiros de Cardiologia, 113(3), 429-434.

Luz, P. L. (2019). Telemedicina e a Relação Médico-Paciente. Arq. Bras. Cardiol., 113(1), 100-102.

Machado, F. S. N., Carvalho, M. A. P., Mataresi, A. et al. (2010). Utilização da telemedicina como estratégia de promoção de saúde em comunidades ribeirinhas da Amazônia: experiência de trabalho interdisciplinar, integrando as diretrizes do SUS. Ciência \& Saúde Coletiva 15(1), $247-254$.

Maldonado, J. M. S. V., Marques, A. B. \& Cruz, A. (2016). Telemedicina: desafios à sua difusão no Brasil. Cadernos de Saúde Pública, 32, e00155615,

Mattos, S. S, Hazin, S. M. V., Regis, C. T. et al. (2015). A telemedicine network for remote paediatric cardiology services in north-east Brazil. Bulletin of the World Health Organization. 93,12: 881-7.

Paula, A. C., Maldonado, J. M. S. V. \& Gadelha, C. A. G. (2020). sHealthcare telemonitoring and business dynamics: challenges and opportunities for SUS. Revista de Saúde Pública. 54, 65.

Sabbatini, R. M. E. (2012). A telemedicina no Brasil: evolução e perspectivas. Informatica em Saúde: Uma Perspectiva Multiprofissional dos Usos e Possibilidades. São Caetano do Sul Yendis Editora.

Santos, W., Júnior, J. H. S., Soares, J. C. et al. (2020). Reflexões acerca do uso da telemedicina no Brasil: Oportunidade ou ameaça? Revista de Gestão em Sistemas de Saúde-RGSS.

Silva, R. S., Schmtiz, C. A. A., Harzheim, E. et al. (2021). O Papel da Telessaúde na Pandemia Covid-19: Uma Experiência Brasileira. Ciência \& Saúde Coletiva. 26(6). https://doi.org/10.1590/1413-81232021266.39662020.

Wen, C. L. (2015). Telemedicina e Telessaúde: Oportunidade de novos serviços e da melhoria da logística em saúde. Panor Hosp, 24-6. https://telemedicina.fm.usp.br/portal/wpcontent/uploads/2015/03/03132015_Revista_Panorama_Hospitalar_Fev_2015_pag24a26.pdf

World Health Organization. (2017). Global diffusion of eHealth: making universal health coverage achievable: report of the third global survey on eHealth. World Health Organization, 2017.

Zanaboni, P., Knarvilk, U. \& Wootton, R. (2014). Adoption of routine telemedicine in Norway: the current picture. Global health action, 7, 22801. https://doi.org/10.3402/gha.v7.22801

Zanotto, B. S., Etges, A. P. B. S., Siqueira, A. C. et al. (2020). Avaliação Econômica de um Serviço de Telemedicina para ampliação da Atenção Primária à Saúde no Rio Grande do Sul: o microcusteio do Projeto TeleOftalmo. Ciência \& Saúde Coletiva, 25, 1349-1360. 